\title{
L'épochè de la parole (la parole soustraite de Heidegger)
}

Vincent Houillon

\section{(2) OpenEdition}

1 Journals

Édition électronique

URL : http://journals.openedition.org/alter/1401

DOI : 10.4000/alter.1401

ISSN : 2558-7927

Éditeur :

Association ALTER, Archives Husserl (CNRS-UMR 8547)

\section{Édition imprimée}

Date de publication : 1 octobre 2011

Pagination : 141-155

ISBN : 978-2-9522374-7-5

ISSN : $1249-8947$

\section{Référence électronique}

Vincent Houillon, «L'épochè de la parole (la parole soustraite de Heidegger) », Alter [En ligne], 19 | 2011, mis en ligne le 01 janvier 2020, consulté le 23 janvier 2020. URL : http://journals.openedition.org/alter/ 1401 ; DOI : 10.4000/alter.1401 


\section{L'ÉPOCHÈ DE LA PAROLE (LA PAROLE SOUSTRAITE DE HEIDEGGER)}

Vincent Houillon

"... ne rendre à l'aventure que des sons vagues et profonds, devenir un arbre que le vent étreint et berce, c'est un but vers lequel on peut s'efforcer; par le plus humain des moyens qui est le langage; être sorti de l'humanité et préférer des sons de feuilles, de gong, d'avalanches, sortir de l'humanité et la surplomber, la couvrir de son ombre, la couvrir de son bruit, l'enfouir sous son feuillage, cela est digne d'efforts ", Pierre Michon, Corps du roi.

\section{La soustraction de la parole}

$\mathrm{Au}$ Dasein, il ne manque certainement pas la parole, même si le phénomène de la parole ne reçoit pas de la part de Heidegger tout d'abord dans Etre et Temps l'analyse qu'il consacre aux existentiaux fondamentaux : le phénomène de la parole est fondé sur la compréhension de l'être et l'affection. Pourtant le parler qui le fonde est " existentialement cooriginaire avec l'affection et le comprendre ${ }^{1}$. Seulement l'analyse de la parole ne se déploie qu'au § 34 , après que Heidegger a analysé l'affection et le comprendre, après même le $\S 33$ où est analysé le phénomène de l'explicitation comme appropriation de la compréhension, phénomène pourtant postérieur à l'articulation de la compréhension qu'est le parler. Etre et Temps a commencé ainsi par une certaine soustraction de la parole, ou du moins par une relative soustraction méthodologique de la parole pour ne la faire apparaître comme thème qu'au $\S 34$ :

Que la parole ne devienne que maintenant notre thème, cela doit indiquer que ce phénomène a ses racines dans la constitution existentiale de

1. Heidegger, Être et Temps, §34, p. 129 [161], trad. E. Martineau, Authentica, 1985, désormais cité E.T. 
l'ouverture du Dasein. Le fondement ontologico-existential de la parole est le parler. De ce phénomène, nous avons fait un constant usage au cours de nos interprétations de l'affection, du comprendre, de l'explicitation, de l'énoncé, et pourtant nous l'avons en même temps pour ainsi dire, soustrait à l'analytique thématique².

Etre et temps repose-t-il sur une certaine différance de l'analyse de la parole, d'une retenue de la parole pourtant cooriginaire dans le parler? Et que peut-on apprendre de cette différance de l'analyse de l'articulation alors que sans elle il n'y a pas de compréhension ? Cette retenue n'est-elle qu'une retenue méthodologique, conséquente d'une présentation soumise à la logique pour laquelle l'articulation du phénomène de la compréhension ne peut se produire qu'après l'analyse thématique de la compréhension (et de l'affection) ? Est-ce parce que ce phénomène se soustrait provisoirement à la thématisation avant d'y être soumis par défaut ? Cette retenue est-elle seulement l'expression d'une logique de l'exposition ou l'indication d'un problème plus important dans la question de la parole? La soustraction méthodologique est-elle une manière de conserver ou de garder intact quelque chose de la parole contre ses expressions réductrices de son usage quotidien ou de sa thématisation théorique ? Ou la thématisation théorique ne provient-elle en second lieu que pour montrer tout d'abord, à propos de la parole, que la thématisation est toujours seconde et dérivée de l'usage qui le précède, que la Vorhandenheit est une modification de la Zuhandenheit - en cela conforme à la démonstration de Heidegger ? Et alors la soustraction est plutôt l'indice de la primauté ontologique $d u$ phénomène $d u$ parler, $d$ 'une fondation originaire de la parole! Plutôt que de reprendre et d'exposer à nouveau cette analyse de la parole ${ }^{3}$, nous voudrions mettre l'accent sur cette retenue de l'analyse, sur cette différance de l'analyse et de la thématisation - comme si la parole se refusait à sa thématisation, comme si elle se retenait contre sa représentation ou thématisation et indiquait par là un phénomène plus originaire de la parole : que la parole est d'abord d'usage avant d'être thématisée, que l'être de la parole est dans son usage avant que d'être exposé dans une thématisation théorique qui prendrait la langue comme objet dans une réflexion seconde. Et que cette subordination est fondée sur une retenue plus essentielle encore. Nous voudrions rester auprès de cette retenue de la parole et nous demander si, auprès de cette retenue, nous ne pourrions pas apprendre quelque chose de la parole ou du parler.

\footnotetext{
2. Ibid., p. 129 [160-161].

3 . Cette analyse est non seulement magistralement ré-exposée dans l'article de Françoise Dastur ici-même dans "Heidegger et la question de l'“ essence" du langage » mais aussi prolongée vers la question de son déploiement dans les textes tardifs de Heidegger.
} 
Ainsi au delà de cette première manifestation du parler dans sa soustraction (la soustraction est le premier phénomene du parler avant ou, plus précisément, à même son phénomène quotidien de la parole), nous voudrions saisir le phénomène de la parole là où ce phénomène vient à disparaître et à manquer. La soustraction de l'anayyse de la parole est la première manifestation d'une série de disparitions de la parole. En cela fidèle à Heidegger pour lequel le phénomène d'être advient là où il disparaît... Après l'analyse de la parole et de la langue, il y a un autre passage où le phénomène de parole en vient à être mentionné au cœur de ce qui engage le Dasein à soi-même et à son être comme être-au-monde ; un passage où la parole vient à manquer et, en se manquant, se révèle, passage où de son usage constant qui soustrayait la parole à la thématisation nous pourrions la thématiser dans le retrait de son usage, là où elle n'est plus en usage, là où il n'y a plus d'usage de la parole : dans l'angoisse !

\section{Le « souffle coupé » de l'angoisse}

C'est par le passage étroit de l'angoisse que nous pouvons aborder un rapport authentique à la parole dans sa mise hors d'usage. En révélant l'être du Dasein, l'angoisse manifeste le sens d'être de la parole en tant qu'elle est une structure de l'être-au-monde. Pris dans l'angoisse, le Dasein "flotte en suspens ", il est en suspens dans ce flottement vertigineux. Le suspens nous livre l'être de l'étant ainsi que l'être de la parole : la suspension néantisante suspend la parole, la parole révèle son être dans le suspens. L'angoisse "coupe le souffle $»^{4}$, coupe la parole. Ainsi dans la conférence $Q u^{\prime} e s t-c e$ que la métaphysique, cette coupure de la parole est plus explicitée :

\section{L'angoisse nous coupe la parole. Parce que l'étant glisse dans son ensemble et qu'ainsi justement le Néant nous accule, tout proposition qui énoncerait "l'être " (dirait le mot est) se tait en sa présence. S'il est vrai que dans l'oppression de l'angoisse nous cherchons souvent à combler précisément le vide du silence par un discours au hasard, ce n'est encore là qu'un témoignage pour la présence du néant ${ }^{5}$.}

Dans la parole qui parle sans cesse, qui n'est jamais à court ou à bout de paroles, qui veut remplir le vide du silence (et la parole - le bavardage - est souvent ce remplissement là, remplissement inauthentique), dans une production effrénée de mots, de propositions, de discours, l'abondance de paroles n'implique pas un éclaircissement de la compréhension de l'être, mais plutôt un obscurcissement de son

\footnotetext{
4. E.T., § 40, p. 186.

5. Heidegger, Questions I, «Qu'est-ce que la métaphysique? », Paris, Gallimard, 1968, p. 59.
} 
ouverture dans une clarté trouble et voilante. L'énoncé se porte toujours sur quelque chose, sur un étant : dire, c'est dire quelque chose à propos de quelque chose. Parler, c'est parler sur, causer de, se rapporter à une cause, à une chose qui est l'objet de la discussion. Mais la parole est alors toujours la parole de l'étant dans le manque de l'être. Non pas qu'il ne manque à l'être que la parole mais qu'il ne manque à la parole que l'être. L'être : le manquant de la parole que toute parole manque à dire. Manque à dire, $c^{\prime}$ est-à-dire tend à dire dans son manque et comme manque, sans toutefois que ce manque soit à nouveau compris comme un étant, une privation de l'étant ou un étant manquant. La parole qui énonce le est de l'étant semble le révéler dans l'énoncé, dans la proposition prédicative, mais en réalité l'obscurcit en l'utilisant en vue de ses propres fins et intérêts. Ce que le manque de la parole dans l'angoisse nous révèle, c'est l'inadéquation de la prédication, de l'énoncé pour dire l'être - et c'est la parenté essentielle de la parole et du néant ${ }^{6}$. L'être se dit autrement que sous la forme de la prédication - dans le suspens de la prédication et de la structure énonciative ou prédicative. Pourtant, comme nous le rappelions plus haut, "le parler est l'articulation de la compréhensivité ${ }^{7}$, la parole est l'articulation de l'intelligence du monde. Dans la parole - dans l'énoncé et l'explicitation - le sens est déjà articulé : l'enchaînement de l'articulation désigne la signification qui, comme l'articulé de l'articulable, revêt toujours du sens. Puisque la parole est l'articulation de la compréhension du là, elle est un existential original de l'ouverture, et a aussi un rapport au monde selon la structure de renvoi. La parole est alors l'articulation signifiante de la compréhension de l'être-au-monde, qui s'exprime dans des mots irréductiblement signifiants.

\section{Le « suspens accentuel»}

La structure de renvoi constitutive du sens du monde fonde celle de la théorie du signifiant qui renvoie à un autre signifiant (la structure existentiale fonde la structure linguistique du signifiant) : $c^{\prime}$ est la « logique du signifiant $»^{8}$. Le signifiant est un étant qui a pour

\footnotetext{
${ }^{6}$. Giorgio Agamben, Le langage et la mort, Paris, Christian Bourgois, 1991.

7. E.T., §34, p. 161.

${ }^{8}$. Nous partirons de Lacan et de sa reprise ou de son «détournement» de la linguistique de Ferdinand de Saussure, comme le nomment Philippe Lacoue-Labarthe et Jean-Luc Nancy, Le Titre de la lettre. Une lecture de Lacan, Paris, Galilée, 1990. Ils reprennent cette logique du signifiant à Lacan lui-même en renvoyant à un article de J.-A. Miller, "La suture. Eléments pour une logique du signifiant ", Cahiers pour l'analyse, no 1, janv-avril 1966, p. 39-51. Nous citons ce texte aussi pour le commentaire de la lecture lacanienne de Heidegger que les auteurs traitent dans leur dernier chapitre "la vérité "homologuée" », ibid., p. 171-189.
} 
être de renvoyer à un autre signifiant. La vérité qui se signifie relève alors du signifiant, dont le propre est de n'avoir de sens que dans un rapport à un autre signifiant, suivant la structure de renvoi de la mondanéité. Comme le souligne Lacan, le signifiant est l'attente du prochain signifiant, il possède une structure temporelle qui est comme celle du dépassement anticipant ${ }^{9}$ de l'arrière (le début de la phrase) vers l'avant (la fin de la phrase). Le sens est le rapport des signifiants entre eux. Mais quelle est l'essence de ce rapport ? Quelle peut être la rencontre du pur rapport des signifiants hors du renvoi indéfini d'un signifiant à un autre, selon sa structure de renvoi ? La rencontre de ce pur rapport des signifiants entre eux ne peut se faire que dans le silence, que dans ce que Alain Juranville, dans sa lecture de Lacan, appelle le « suspens accentuel »10. Dans la suspension de la parole, dans le coupé du souffle, dans la suspension néantisante du bavardage se rencontre le pur rapport des signifiants : l'être ou la vérité $^{11}$. La coupure des signifiants ou l'arrêt suspensif du renvoi des signifiants sont éprouvés comme le manque des signifiants - comme le manque, non de quelques-uns, mais de tous les signifiants. Ceux-ci, selon leur structure temporelle $d^{\prime}$ anticipation, indiquent toujours la nécessité du reste à venir de la phrase. Dans le silence, la vérité totale est aperçue : le silence est l'aperception originale de l'être même. L'être se révèle dans l'arrêt du renvoi indéfini des étants aux autres étants. L'être coupe le souffle dans l'angoisse. Le souffle est l'entrain qu'on a à parler, à toujours produire des mots. Le souffle, c'est

\footnotetext{
9. Cf. Alain Juranville, Lacan et la philosophie, Paris, P.U.F, 1988, «Conscience et monde. Anticipation et signe » p. 28-39. Ce livre est la lecture la plus complète de la relation de Lacan aux philosophes. En ce qui concerne le rapport à Heidegger, cf. "L'émergence du signifié, le sujet et l'Autre ; Lacan et Heidegger », ibid., p. 128-140.

10. «La vérité totale du signifiant pur», ibid., p. 377-388. Le suspens accentuel désigne l'articulation en suspens entre deux propositions analysées depuis la structure trinitaire du réel, du symbolique et de l'imaginaire : «Le propre du suspens accentuel, c'est que les trois y sont posés ensemble, "jetés" vers l'à-venir, le réel comme ce qui est à l'avance passé, le symbolique comme la coupure toujours à venir du présent, l'imaginaire comme l'anticipation irréductible $\mathrm{du}$ futur. Nouées ensemble dans le suspens accentuel, les trois ex-stases constitutives du temps sont prises dans une anticipation qui caractérise l'imaginaire, l'ouverture même du temps. Le temps apparaît donc comme essentiellement positif. Il est d'abord l'acte d'anticipation qui pose le signifiant dans sa signifiance et le fait advenir. Sans cet acte d'anticipation, il n'y aurait pas d'ouverture du temps et de jet des trois ex-stases du passé, du présent et du futur ». Ibid., p. 378 ; pour une explication avec Heidegger de la différence entre le futur et l'avenir, voir plus loin p. 383.

11. La suspension de la parole ou le silence constitue, avec le bavardage, un couple comparable au couple de concepts de la parole pleine et de la parole vide chez Lacan : « La parole pleine, dira Lacan, est celle qui présente au sujet la révélation de sa vérité, qui effectue l'alèthéia, le dévoilement dont parle Heidegger. Mais elle n'est qu'un idéal ». Ibid., p. 114-115. Le suspens est ce qui produit l'apparition du sujet, comme pour nous la suspension épochale de la parole (le silence) révèle l'être de l'homme. Cependant Juranville distingue le sujet de "l'être-au-monde jeté par l'être selon Heidegger » (ibid., p. 384) en tant que le sujet relève de la jouissance pure de l'encore du temps.
} 
l'articulation, la virgule, la pause ou la séparation des énoncés, qui ne laissent pourtant pas entendre l'être, puisque seul l'étant est visé dans l'articulation des signifiants. Le suspens accentuel est comme la conversion du regard de l'étant visé vers la visée elle-même (réduction phénoménologique), comme la reconduction de l'étant vers l'être. Dans la coupure du souffle se révèle l'être. L'être se manifeste comme être dans le souffle coupé.

\section{Le silence et la voix de l'appel de conscience}

L'être doit pourtant être dit. Il est dit dans le mode insigne du dire qu'est le silence. "Pour pouvoir faire-silence, le Dasein doit avoir quelque chose à dire, c'est-à-dire disposer d'une résolution authentique et riche de lui-même. C'est alors que le silence manifeste et brise le «bavardage». Le silence, en tant que mode du parler, articule si originairement la compréhensivité du Dasein, que c'est de lui que provient le véritable pouvoir entendre et l'être-l'un-avec-l'autre translucide. $»^{12} \mathrm{C}^{\prime}$ est dans la garde du silence ${ }^{13}$, dans le silence fait ou gardé, que l'être se dit avec la voix en tant qu'elle est le manque de signifiant, en tant qu'elle ne dit rien. L'être se dit avec la voix qui ne dit rien.

Cette voix est la voix de l'appel qui, comme tel, n'énonce rien, ne donne pas d'informations sur le monde et n'a rien à raconter ou à dire. " Au soi-même ad-voqué rien n'est crié, mais il est con-voqué à lui-même, c'est-à-dire à son être le plus propre $»^{14}$. Dans le fairesilence se fait entendre la conscience (Gewissen) ${ }^{15}$ : le Dasein est convoqué par l'appel auquel il ne peut se soustraire, dont il n'a pas l'initiative, qu'il ne peut maîtriser mais dont il se soucie ${ }^{16}$. Le Dasein

\footnotetext{
12. E.T., p. 165.

13. Dans les textes ultérieurs de Heidegger, le silence sera désigné comme l'origine de la parole dans la "métamorphose du langage", Beiträge zur Philosophie, GA 65, Vittorio Klosterman, Frankfort-sur-Main, 1989, p. 78, dans une « sigétique ». Cf. Jean Greisch, «La parole d'origine, l'origine de la parole. Logique et sigétique dans les Beiträge zur Philosophie de Martin Heidegger ", Rue Descartes, no 1, p. 191-212. Le silence est l'essence du langage. "L'être lui-même, précisément dans la mesure où nous l'atteignons au moyen du saut, nous ne pouvons jamais le dire immédiatement. Car tout dire (Sage) provient de l'être et parle à partir de sa vérité. Toute parole et ainsi tout dire est placé sous le pouvoir de l'être. L'essence de la «logique » est donc la sigétique. C'est seulement en elle qu'est également comprise l'essence du langage ». Beiträge zur Philosophie, GA 65, p. 79.

14. E.T., §56, p. 273.

15. Cf. Jean-François Courtine, «Voix de la conscience et vocation de l'être », dans Heidegger et la phénoménologie, Paris, Vrin, 1990, p. 305-325.

16. Cf. Jacques Taminiaux, "La phénoménologie de l'angoisse dans Sein und Zeit », dans Figures de la Finitude, éd. par G. Florival, Peeters, Bibliothèque philosophique de Louvain, 1988, n 32, p. 1-32. Après avoir rappelé que l'appel de conscience est un « nœud pour les existentiaux fondamentaux que sont la compréhension, le discours, la chéance», Jacques Taminiaux
} 
ne s'appelle pas, l'appel ne peut provenir d'un autre être-au-monde, mais « ça » appelle. Bien que l'appel provienne du Dasein, néanmoins il n'en provient pas et le dépasse : "L'appelant n'est "mondainement" déterminable par rien en son qui. Il est le Dasein en son étrang(èr)eté, il est l'être-au-monde originellement jeté en tant qu'hors-de-chez-lui, il est le "que" nu dans le rien du monde. L'appelant est non-familier au On-même quotidien - quelque chose comme une voix étrangère ${ }^{17}$.

Cette voix qui ne dit rien, ce silence provoqué par l'angoisse, fait surgir la figure de l'étrang(èr)eté du Dasein. Dans l'angoisse, le Dasein quotidien se sent "étrange » mais aussi «étranger», chassé de son chez-soi. L'angoisse brise la caractérisation formelle du Dasein comme chez soi. L'angoisse, qui jette le Dasein devant son être-propre, le ramène à l'impropriété de sa propriété, à son archi-propre: le hors-dechez-soi. L'archi-propre n'est pas la cause du propre, mais ce qui régit le propre dans sa propriété. L'étrang(èr)eté est l'impropre du propre : non l'impropre du Verfallen du Dasein, mais l'im-propre co-originaire du propre. L'être-au-monde, dans son existence quotidienne, dans l'assurance d'un chez-soi habitable, a son fondement dans l'étrange inquiétude ou l'inquiétante étrangeté du Dasein. Le hors-de-chez-soi, l'ex-propriation constitue essentiellement l'être du Dasein, son soi d'étrang(èr)eté ${ }^{18}$, son soi de transcendance dans la retenue du néant. Qu'est-ce que signifie le Dasein depuis la révélation de son archipropre, depuis la mise entre parenthèses (épochè) des déterminations métaphysiques de l'homme?

implique à nouveau l'existential de la Befindlichkeit, dont il dit que « l'analyse pèse sur lui lorsqu'elle interprète existentialement l'appel de conscience à la lumière du souci. Relevons à cet égard trois questions capitales qui dominent cette interprétation : Qui appelle ? A quoi l'appel engage-t-il ? Qu'est-ce que comprendre cet appel ? Heidegger répond que dans l'appel de conscience, c'est le Dasein lui-même qui est l'appelant, mais le Dasein entendu dans ce qui lui est fondamental, le souci même que voilent les préoccupations et les sollicitudes quotidiennes. ", ibid., p. 26.

${ }^{17}$ E.T., §57, p. 276-277. Voix étrangère ou voix de l'ami ou voix étrangère de l'ami. Cf. JeanFrançois Courtine, "La voix (étrangère) de l'ami. Appel et/ou dialogue ", dans Heidegger et la phénoménologie, op.cit., p. 327-353. Voir également la lecture de Jacques Derrida de cette voix de l'ami dans Politiques de l'amitié, Paris, Galilée, 1994, p. 343-365.

${ }_{18}$ Cette étrang(èr)eté est celle de l'homme comme le deinotaton de l'Antigone de Sophocle, le plus inquiétant parmi l'inquiétant, dont Heidegger fait la lecture dans L'Introduction à la métaphysique : "Le dict "l'homme est ce qu'il y a de plus inquiétant" donne la véritable définition grecque de l'homme ». Introduction à la métaphysique, Paris, Gallimard, 1967, p.158. L'inquiétude est l'épochè de la figure de l'homme dans l'angoisse réductive, son mouvement de déshumanisation comme nous allons le voir ensuite. 


\section{La mise entre parenthèses de l'homme et le Dasein « réduit »}

Dans l'angoisse est suspendue la validité de la thèse de l'homme avec ses présupposés ontologiques. L'homme, selon ses déterminations positives, selon les présupposés de sa conception métaphysique, est mis entre parenthèses. L'angoisse met l'homme entre parenthèses : elle ne l'aneantit pas, mais elle révèle les structures ontologiques fondamentales sur lesquelles l'homme peut être compris comme un sujet. L'angoisse produit une épochè de la thèse métaphysique de l'homme. L'angoisse est l'épochè de l'homme. L'angoisse suspend la thèse de l'homme comme animal rationnel pour le conduire à une constitution ontologique qui ne présuppose aucune thèse philosophique : elle conduit l'homme au Da-sein. L'angoisse reconduit l'homme au Dasein dans l'homme. Et cette reconduction est bien une réduction de l'homme au Da-sein. Le Dasein est le régime réduit de l'homme. L'angoisse met fin à un concept déterminé de l'homme : l'angoisse marque bien la fin de l'homme 19 et l'irruption du rien du là qui reste. L'angoisse est la fin de l'homme ainsi que sa détermination nonhumaine: le Da-sein serait l'humanité de l'homme, humanité nonhumaine ou a-humaine. L'angoisse amène l'homme à sa fin, elle amène l'homme là où il s'achève ou se suspend: au là de sa possibilité.

Pris dans l'angoisse, le Dasein «flotte en suspens », il est laissé en suspens dans le "glissement de l'étant». Le Dasein comme être-aumonde n'est que cet être-laissé en suspens, même s'il n'est plus dans l'incertitude ou l'indécision, puisque le suspens est la venue essentielle au paraître de la décision co-originaire de l'ouverture. Dans le suspens se dévoile l'être du Dasein. "Seul le pur Da-sein est encore là dans la secousse qui le laisse en suspens, et qui ne lui permet de se raccrocher à rien $»^{20}$. Alors se dévoile l'être-dévoilant du Dasein: le Da-sein est un dévoiler. Mais ce dévoiler se dévoile dans le dévoilement du néant, c'est-à-dire le dévoilement originaire qui laisse être le

\footnotetext{
19. Cf. Jacques Derrida, «Les fins de l'homme» dans Marges, Editions de Minuit, Paris, 1972. Voir également l'intervention de Philippe Lacoue-Labarthe sur ce texte dans "Au nom de... » dans Les fins de l'homme, à partir du travail de Jacques Derrida, Paris, Galilée, 1981, p. 415-438. P. Lacoue-Labarthe, à partir d'une analyse des mêmes passages de Qu'est-ce que la métaphysique?, accentue, à la différence de J. Derrida qui suit l' «aimantation » du texte heideggérien par le motif du propre, une certaine dépropriation de l'homme, en interrogeant la problématique de l'Unheimlichkeit dans la question de l'homme. La suspension que nous suivons à la trace est évidemment la suspension de l'Unheimlichkeit : l'étrangeté est la suspension, ce qui nous maintient en suspens dans l'entre-deux du chez-soi.

20. Questions I, "Qu'est-ce que la métaphysique?», op. cit., p. 59. «Nur das reine Da-sein in der Durchschütterung dieses Schwebens, darin es sich an nichts halten kann, ist noch da». GA 9, p. 112.
} 
dévoiler du Dasein. Le flottement est le flottement du Da-sein ${ }^{21}$. Le Dasein est en suspens dans ce flottement.

L'angoisse révèle le Da-sein à lui même. Dans le glissement de l'étant en totalité, nous les hommes, nous glissons vers notre propre essence, vers le Da-sein "dans» l'homme (comme l'indique une remarque ajoutée en 1949 : das Da-sein » im "Menschen). Dans l'angoisse, il ne reste que le là de l'être-là. Seul le là est encore là, dans le recul de l'étant en totalité et dans le glissement de l'homme. Le là n'est pas un lieu qui appartient au monde ou à la totalité de l'étant, mais il est le lieu du recul de la totalité de l'étant, le lieu où le Da-sein apparaît à lui-même pour la première fois. Il est le lieu de la révélation du Dasein pour lui-même. L'homme disparaît avec la totalité des étants dans son glissement. Ne reste, dans ce glissement de l'homme, que le là de ce glissement, que le là de l'être : le Da-sein. L'angoisse est l'expérience de la disparition de l'homme et de la révélation $d u$ Da-sein. Entendons la disparition comme la mise en suspens de l'homme dans le là de l'être, dans le Da-sein. Nous sommes suspendus dans le Da-sein. Le Da-sein est un être de suspension. La suspension marque le nom même du Da-sein, par le trait d'union qui apparaît entre le da et le sein, entre le là et l'être. Le trait d'union est un trait de suspension. Le rapport unitaire de l'être et de l'homme est la suspension constitutive du rapport de l'être et du Da-(sein). Ce trait de suspension, qui constitue la propriété essentielle du Dasein, manifeste à même le Da-sein l'articulation de l'être et de son avoirlieu, de son appropriation dans la parole ou comme parole. La parole provient de cette suspension du Dasein à l'être ou plutôt de cette suspension de l'être à lui-même dans un suspens originaire que Heidegger ne tardera pas à nommer "épochè » de l'être. Le lieu de l'être est suspendu à l'être : l'être se suspend au lieu de son être ou de son apparaître. Le Da-sein est le lieu de la suspension de l'être. L'époche est la suspension phénoménologisante de l'être qui se phénoménalise au lieu de l'être qu'est le Dasein ${ }^{22}$.

Le Da-sein est un Da-sein suspendu : un Da-sein réduit. Ou plutôt la notion même de Da-sein est sous réduction. Le Da-sein est le Da-sein de la réduction, tout comme l'ego transcendantal est l'ego réduit de la réduction. Cela peut signifier que le Dasein soit l'équivalent du sujet

\footnotetext{
${ }^{21}$. Le flottement est aussi ce qui laisse flotter le Sein et le $D a$ de l'être, l'être et le lieu de la phénoménalisation de l'être : le tiret entre le Da et le Sein du Da-sein indique aussi le lieu sans lieu, le lieu d'un écart entre le lieu de l'être et l'être lui-même. La question du flottement est aussi la question du lieu de la phénoménalisation de l'être : le Da-sein. Laissons encore flotter notre attention sur ce flottement avant même de le saisir et de l'arrêter.

22. Cf. notre thèse, Le souci de l'épochè, Heidegger et le sens multiple de l'épochalité, dont l'objectif principal consistait à saisir le trait (d'union) de l'épochè de l'être et de l'épochè du Da-sein. Cet article est un extrait remanié de la thèse.
} 
transcendantal mais il ne l'est qu'à la condition expresse de dégager le transcendantal de l'eidétique. Toute l'analytique du Da-sein est opérée sous la réduction à condition de la distinguer de la réduction husserlienne des positions objectivantes qui découvre un « résidu » opérateur de ces validations objectivantes, résidu dont le mode d'être serait entendu depuis le mode d'être de l'étant-sous-la-main. La réduction ici visée n'est pas la réduction eidétique mais celle qui reconduit à l'être du Dasein puis au sens d'être lui-même. L'homme est reconduit à l'être et est ainsi compris comme l'être dont l'être est le sens d'être : Da-sein. Le $D a$-sein est un "être réduit », conduit sous la réduction de la thèse (métaphysique) de l'homme. Et cette réduction est la suspension du néant ou l'homme est re-conduit au Da-sein. L'angoisse dégage la constitution ontologique du Da-sein. Elle est l'expérience fondamentale de l'analytique du Dasein en opérant tout d'abord une destruction de l'interprétation métaphysique de l'homme sous la forme d'une déshumanisation méthodologique de l'homme.

\section{La déshumanisation}

Grâce à l'analyse de l'angoisse, il semble que l'on retrouve la thématique essentielle de la déshumanisation proposée par Fink dans le dégagement du spectateur phénoménologique qui apparaît dans l'exercice de l'épochè et dans l'opération réductive. L'inhibition du monde naturel, des rapports mondains qui soutiennent la croyance et les positions thétiques de monde, se révèle comme le glissement du monde selon le recul des étants manifestés dans l'angoisse. Fink découvre alors la tendance transcendantale qui s'éveille en l'homme: celle-ci est l'éveil (Weckung) en l'homme de ce qu'il est incapable, comme homme, de produire en soi et à partir de soi. Ce mouvement qui rapporte l'homme à soi-même est l'eveil en l'homme qui s'éveille à soi :

La tendance transcendantale qui s'éveille en l'homme, qui le pousse à
inhiber d'un seul coup toute validité, supprime (aufhebt) l'homme lui-
même, l'homme se dés-humanise (sich entmenscht) dans l'accomplis-
sement de l'épochè, c'est-à-dire qu'il libère en lui le spectateur trans-
cendantal, il périt en lui. Mais celui-ci n'est pas d'abord advenu par
l'épochè, il s'est seulement affranchi du travestissement voilant de
l'être-homme ${ }^{23}$.

${ }^{23}$ Eugen Fink, Sixième Méditation cartésienne. L'idée d'une théorie transcendantale de la méthode,
Grenoble, J. Millon, 1994, p. 93. Dans la préface, la traductrice, Natalie Depraz, dit que cette dés-
humanisation est une " forme particulière de génétisation de la réduction » (p. 39), qui vient se
substituer à la présentation de la réduction selon sa voie cartésienne dans les Idées directrices de
la phénoménologie. Cette voie reconduisait à une structure oppositionnelle entre la conscience
transcendantale immanente et le monde réel transcendant et, finalement, rendait confuse la
distinction entre l'épochè et la réduction. La voie de Fink confirme la validité des critiques 
L'angoisse ne fait-elle pas apparaître une telle dés-humanisation de l'homme en dévoilant le Dasein dans son authenticité ? Est-ce seulement un dévoilement de l'authenticité ? Ou n'est-ce pas plutôt le dévoilement du Dasein lui-même, de sa structure ontologique et de son rapport constitutif à l'être même qui se dévoile (néant) ? L'angoisse libère le Dasein (au sens de la Freilegung), elle délivre dans le Dasein inauthentique le Dasein authentique. Elle est la délivrance de la structure ontologique du Dasein à l'encontre de ce qui recouvrait le Dasein dans la compréhension de celui-ci comme un homme, comme un sujet subsistant ${ }^{24}$. L'angoisse est la libération du Dasein de l'homme, l'affranchissement des entraves pesant sur le Dasein inauthentique où le plus souvent le Dasein existe dans la crispation et le cramponnement à l'étant. La libération du Dasein est la sauvegarde retrouvée de l'authenticité du Dasein : le Dasein se libère de l'homme. L'angoisse laisse être le Dasein en l'homme. L'angoisse est le dévoilement du Dasein qui est usuellement recouvert par l'homme constitué comme couche psychique, comme composé d'une âme et d'un corps, comme sujet substantiel. Elle laisse se produire le dévoilement du Dasein recouvert par le Dasein inauthentique, c'est-à-dire par le Dasein qui se comprend à partir des étants du monde où il se mire, qui se perd dans le mirement du Dasein depuis sa réflexion optique. L'homme, dans sa détermination métaphysique, apparaît, depuis la révélation de l'angoisse délivrante, comme le recouvrement ou le voilement du Dasein, tout comme il l'est, pour Fink, de la subjectivité transcendantale : «Ce n'est pas l'homme qui fait réflexion sur son soi, mais c'est la subjectivité transcendantale, voilée dans l'auto-objectivation sous la forme de l'homme, qui fait réflexion sur elle-même, en prenant son départ apparemment comme homme, en

\footnotetext{
qu'Heidegger avait adressées à Husserl dès 1925. La voie de la déshumanisation indique en creux la "répétition " heideggérienne de la réduction husserlienne dans sa distinction avec l'épochè par sa propre mise en œuvre d'une radicalisation de la réduction phénoménologique.

${ }^{24}$. Denise Souches-Dagues remarque que «ce qui tient lieu chez lui [Heidegger] de réduction, c'est la libération (Freilegung) de la question de l'être : un laisser-être, et non un retournement réflexif qui risquerait de pervertir le mode d'être du Dasein, sa disposition la plus prochaine ». «La Lecture husserlienne de Sein und Zeit », Notes sur Heidegger, Paris, Editions de Minuit, 1993, p. 127. Mais l'angoisse ne présente-t-elle pas, tout comme la mort, une ré-flexion antérieure à la réflexion? Fink présente la réflexion comme la réflexion de la subjectivité transcendantale sur elle-même et non pas comme une réflexion de l'homme. L'épochè est différente de la réflexion sur les actes d'une conscience intentionnelle. La réflexion n'est pas encore l'épochè, qui est seule le moment de la suspension, du recul des étants amorçant leur conversion vers le mode d'être. Nous tentons de viser ici une ré-flexion ontologique, préalable à la réflexion de la conscience représentative, par laquelle le Dasein fait retour sur soi dans un mouvement de retour qui est la découverte fondamentale de son soi. Ce n'est pas une réflexion de l'entendement, mais un retournement du Dasein sur lui-même. Nous avons ailleurs tenté de montrer que la mort, dans son rapport intime avec l'angoisse, constitue une réflexion absolue : la réflexion mortelle est pré-réflexive, antérieure à la réflexion au sens de la réflexion d'entendement. Cf. «Le fondement mortel de la phénoménologie », ALTER, n 2, 1994, p. 313-332.
} 
se dépassant et se ruinant comme homme, à savoir en s'orientant vers le fond vital (Lebensgrund) propre, en dernière instance le plus intime $»^{25}$. L'angoisse est la ruine de l'homme dans son orientation vers l'être pour lequel le Dasein s'angoisse. Et cela même si l'orientation n'est pas celle qui se produit sur le fond vital, puisque ce n'est pas proprement la vie du Dasein qui est dévoilée mais son existence, son ek-sistence vers l'être ${ }^{26}$. L'angoisse s'angoisse pour l'être du Soi, pour l'être du Dasein. Puis, dans un deuxième temps, l'angoisse est l'angoisse de l'être. L'angoisse pour son être propre se convertit en angoisse pour le propre de l'être, pour l'être du soi, qui est le fondement de l'être sur le soi : l'être se fonde dans le fondement du Dasein.

\section{La déshumanisation épochale de la parole}

L'angoisse s'identifie donc à la réduction comme reconduction à l'être de l'étant, dans le glissement de l'étant et dans la révélation de la transcendance. Elle est la réduction de l'homme au Da-sein, la reconduction de l'étant-homme à l'être même de l'homme : au $D a$ sein. Le Da-sein dans l'homme, c'est l'être de l'homme. L'analytique du Dasein est donc bien cette reconduction de l'étant-homme à l'être de l'homme, que l'angoisse révèle comme Da-sein. Cette réduction provient du néant, de l'essence de l'être. L'être même "opère » la réduction qui reconduit l'étant à l'être, et cette opération est l'angoisse elle-même. La déshumanisation angoissante accomplit la radicalisation de la réduction phénoménologique. Mais l'angoisse estelle la seule déshumanisation possible ? Ecartant provisoirement les autres tonalités fondamentales, qui sont autant de reconductions radicales de l'étant à l'être, autant de réductions phénoménologiques ou autant de processus de déshumanisation, nous voudrions indiquer une déshumanisation encore plus originale et plus originaire. Heidegger la présente sans l'appeler expressément ainsi, mais en la parant de tous ses caractères: il s'agit de l'épochè originaire de la parole. Heidegger qualifie la Sprache comme la déshumanisation originaire, en tant qu'elle est une mise entre parenthèses de la représentation de

\footnotetext{
25. Eugen Fink, op.cit, p. 86.

26. Si l'angoisse heideggérienne, dans son esseulement, permet le dévoilement du Dasein comme être-au-monde, analogue à la mondanéité transcendantale de l'ego constituant, l'isolement chez Fink est l'isolement du noyau spectatoriel, il est un isolement du monde, de la mondanéité naturelle comme de la mondanéité transcendantale, au sens où l'ego transcendantal est constitutif du monde. Cet isolement apparaît alors comme une "retraite hors du monde, hors de tout procès d'auto-objectivation humaine et mondaine " (Préface par Natalie Depraz de E. Fink, op.cit., p. 39). Mais il resterait un autre sens possible de cet isolement : le refus du monde apparaîtrait comme le refus que ne peut constituer le Dasein. Le Dasein est un être-au-monde depuis le refus du monde, depuis le monde qui se refuse à son propre pouvoir de constitution. Cf. notre article, « Le refus du monde : Heidegger après le tournant » dans Alter nº 6, 1998.
} 
l'homme comme d'un étant subsistant, comme d'un être vivant compris selon l'ordre de la subsistance ou de l'étant-sous-la-main. Ainsi dans les Contributions à la Philosophie écrit-il :

La parole, parlée ou tue, est la première et la plus vaste humanisation de l'étant. Semble-t-il. Mais elle est précisément la déshumanisation la plus originaire de l'homme comme être vivant sous la main ou "sujet » et de tout ce qui a été jusque là. Et ce faisant [elle est] la fondation de l'existence et de la possibilité de la déshumanisation de l'étant ${ }^{27}$.

La première partie de la phrase, avant son renversement de la thèse dénoncée comme apparente, affirme pourtant bien le contraire d'une déshumanisation de la parole. Elle correspond au développement que Heidegger a consacré à la thématique de la déshumanisation dans sa lecture de Nietzsche à la même époque. Car en réalité le thème de la déshumanisation est spécifiquement travaillée par Heidegger dans son interprétation de Nietzsche dans les cours de 1936-1937 ou il exprime ses « réserves » au sujet de l'humanisation de l'étant, au sujet de l'introduction de l'homme dans l'étant: il est évident que toute interprétation de l'étant est toujours une interprétation humaine et se situe dans son rapport. Il évoque alors la parole comme une manière d'humaniser l'étant: "Même toute parole adressée à l'étant, toute dénomination de l'étant par le langage est une façon de recouvrir l'étant d'une construction humaine, de capturer l'étant dans de l'humain, dès lors que le mot et le langage illustrent suprêmement l'être de la nature humaine ${ }^{28}$. Pourtant il n'entend pas invalider la possibilité d'une déshumanisation de l'étant en perçant l'aporie ou l'impasse de l'humanité dans toute tentative humaine, serait-ce celle de la déshumanisation, à la condition expresse d'interroger à nouveau l'essence de l'homme. Car la "question demeure de savoir si la détermination de l'essence de l'homme humanise celui-ci ou le déshumanise. La possibilité subsiste, que l'accomplissement de la détermination de l'essence de l'homme demeure toujours et nécessairement l'affaire de l'homme, et qu'elle soit, pour autant humaine, mais que la détermination même, sa vérité, élève l'homme hors et audelà de lui-même et de la sorte le déshumanise, et du même coup attribue une autre essence à l'humain accomplissement de la détermination de l'essence de $l^{\prime}$ homme ${ }^{29}$. Si la percée est possible, elle s'annonce dans ce retournement concernant la parole exprimé dans les Contributions : processus d'humanisation, en apparence, la parole

\footnotetext{
27. „Sprache, ob gesprochen oder geschwiegen, die erste und weiteste Vermenschung des Seienden. So scheint es. Aber sie gerade die ursprüngliche Entmenschung des Menschens als vorhandenes Lebewesen und "Subjekt" und alles Bisherigen. Und damit Gründung des Da-seins und der Möglichkeit der Entmenschung des Seienden". Beiträge zur Philosophie, GA 65, § 281, Die Sprache (ihr Ursprung), p. 510.

28. Heidegger, Nietzsche I, Paris, Gallimard, 1971, p. 280.

29. Ibid., p. 282-283.
} 
est dans son essence déshumanisation, à condition de l'entendre originairement depuis l'être et plus spécialement depuis l'épochè de l'être.

La parole accomplit originairement une épochè des thèses métaphysiques qui constituent l'homme dans un ordre non proprement humain. La parole est une sorte d'épochè spontanée et originaire, antérieure à toute épochè et réduction s'accomplissant dans la méthode de la phénoménologie ${ }^{30}$. La réduction phénoménologique, même radicalisée, est enracinee dans l'Entmenschung originaire de la parole qui permet de déployer une relecture de la réduction comme reconduction à l'être depuis la parole elle-même ${ }^{31}$. Heidegger y effectue son propre linguistic turn, son propre tournant de la parole dans la découverte de l'essence de la parole comme épochè.

Car c'est là la découverte la plus étonnante de Heidegger concernant l'essence de la parole, et cette découverte apparaît comme phénoménologique si l'on comprend authentiquement le sens de l'épochè de l'être comme An-sich-halten. L'être se déploie dans et en tant qu'il se retient (an sich hält). "L'être même s'essencifie en tant que le se-retenir soi-même $»^{32}$. Et dans cette retenue l'être se soustrait au dévoilement, maintenant le voilement dont s'origine le dévoilement et la manifestation de l'étant qui advient dans et par une telle soustraction. L'être se promet à lui-même dans le dé-voilement de l'étant et cette promesse forme la parole originaire. Voici ce qu'il dit de l'essence de la parole dans « Le déploiement de la parole »:

Plus d'une raison parle en faveur du fait que c'est précisément le déploiement de la parole qui lui interdit (verweigert) de venir à la parole - à cette parole en laquelle nous formulons des énoncés sur la parole. Si la parole interdit en ce sens partout son déploiement, alors cet interdit (diese Verweigerung) fait partie du déploiement de la parole. Ainsi la parole ne se contient-elle pas seulement là où nous la parlons à la façon

\footnotetext{
30. Fink également parle d'une «réduction de la langue » depuis la réduction de l'idée d'être corrélative de la réduction phénoménologique. Op. cit., p. 149. Husserl refuse, quant à lui, une telle réduction de la langue : «Le sens naturel de la langue ne doit pas être transféré au transcendantal par une réduction que l'on exerce sur lui ». Op. cit., Notes Manuscrites, p. 149. Voir également l'introduction de la traductrice Natalie Depraz, "Une langue transcendantale ? " ibid., p. 43-48. Mais la découverte de Heidegger ne serait pas tant celle d'une réduction de la langue que celle de la langue dans son essence comme réduction. Notons que Fink définit, lui aussi, l'activité phénoménologisante du spectateur phénoménologique comme "sprachlos ", "dénuée de langue », ibid., p. 151.

31. L'idée d'une réduction phénoménologique comme parole, ou enracinée dans la parole ou la langue, a été l'un des enjeux de la déconstruction derridienne de la phénoménologie, notamment dans sa lecture de la réduction husserlienne, de la pré-réduction spontanée dans le pouvoir même de l'expression qui va à l'encontre de l'intention expresse de Husserl, mais qui en est la "vérité » pour Derrida. Et son enjeu, comme le répète R. Bernet, " consiste à donner une forme linguistique à la réduction phénoménologique ou plutôt à montrer son enracinement dans le (ou dans une certaine idée du) langage ». La vie du sujet, Paris, PUF, 1994, p. 276.

32. Nietzsche II, Paris, Gallimard, 1971, p. 307 : « Das Sein selbst west als diese Ansichalten ».
} 
coutumière; cette retenue (An-sich-halten) est déterminée par ceci que la parole se retient et se contient (an sich hält) avec son origine [retenant et réservant son origine], et ainsi refuse (versagt) de dire son déploiement à notre manière habituelle de penser qui est la représentation ${ }^{33}$.

L'épochè, non nommée, tue dans la parole, appartient à l'essence de la parole elle-même épochale! Et la parole se retenant avec son origine indique l'époche dont elle provient et surgit : l'épochè de l'être. La parole s'origine dans la retenue ou épochè de l'être, dans l'être en son retrait et sa donation comme articulation de la parole, selon une articulation elle aussi plus originaire ${ }^{34}$ que l'articulation de la compréhension dans Être et Temps.

Dans Etre et Temps la différance de l'analyse de la parole était certes méthodologiquement fondée sur la primauté de la Zuhandenheit sur la Vorhandenheit mais plus essentiellement celle-ci provient de l'essence de l'être comme retenue, comme épochè, comme être qui se refuse (verweigert) dans un refus (Verweigerung) essentiel que Heidegger aura déployé depuis les Contributions à la philosophie. La soustraction méthodologique provisoire reposait essentiellement sur une soustraction originaire de l'être qui se retient dans le silence et qui advient dans la parole ou comme parole articulée. L'essence de la parole se refuse à la parole et ce refus ou cette épochè garde le secret de la parole, maintient la parole au secret tout comme elle en permet sa dispensation et son histoire. La soustraction de la parole est le silence qui fonde la parole ${ }^{35}$.

\footnotetext{
33. Acheminements vers la parole, Paris, Gallimard, 1976, p. 170.

34. Dans les Beiträge, à la suite de la précédente citation, Heidegger écrit : „Und so ist die Sprache Mab-setzung im Innersten und Weistesten, Mab-setzung als Erwesung des Fugs und seiner Fügung (Ereignis)“. Ibid., p. 510. Cet ajointement (Fügung) est une articulation originaire de la multiplicité, une structuration des moments du déploiement temporel de l'être qui se donne en son retrait (épochè) ou encore la mesure, au sens de la Dikè grecque, du « conflit du monde et de la terre » („Und sofern die Sprache Grund des Da-seins, liegt in diesem die Mäbigung und zwar als der Grund des Streites von Welt une Erde", ibid.).

35. «Die Sprache gründet im Schweigen », ibid.
} 